\title{
II. On the action of the rays of the solar spectrum on vegetable colours, and on some new photographic processes
}

\author{
Sir John F.W. Herschel Bart. K.H. F.R.S.
}

To cite this article: Sir John F.W. Herschel Bart. K.H. F.R.S. (1843) II. On the action of the rays of the solar spectrum on vegetable colours, and on some new photographic processes, Philosophical Magazine Series 3, 22:142, 5-21, DOI: 10.1080/14786444308621625

To link to this article: http://dx.doi.org/10.1080/14786444308621625

电 Published online: 01 Jun 2009.

Submit your article to this journal $\pi$

Џll Article views: 3

Q View related articles $\sqsubset$ 
ceedingly striking, by discharging the steam obliquely as in fig. 5 , in which case the ball will take up its position at a greater distance from the orifice, but will still be sustained in the current, notwithstanding that gravity in this instance acts at an angle to the jet. A hollow globe made of thin brass or copper and from two to three inches in diameter, answers very well for the purpose, where the steam is discharged from an aperture not less than $\frac{1}{2}$ th of a square inch in area.

In the well-known experiment of supporting a ball upon the summit of a jet of water, the ball merely reposes in the hollow formed by the liquid in the act of turning over to fall to the ground, which is very different from being sustained in the current as it is in the case of the steam.

Jesmond Dene, Newcastle-upon-Tyne, WM. Geo. Armstrong. Nov. $15,1842$.

II. On the Action of the Rays of the Solar Spectrum on Vegetable Colours, and on some new Photographic Processes. By Sir John F. W. Hersched, Bart., K.H., F.R.S.*

[With a Plate.]

1497. I N my paper on the "Chemical Action of the Solar Spectrum on preparations of Silver and other substances," read to the Royal Society in February 1840, and of which the present communication is intended as a continuation or supplement, some experiments on the effect of the spectrum on the colouring matter of the Viola tricolor, and on the resin of guaiacum are described, which the extreme deficiency of sunshine during the summer and autumn of the year 1839 prevented me from prosecuting efficiently up to the date of that communication. The ensuing year 1840 was quite as remarkable for an excess of sunshine as its predecessor for the reverse. Unfortunately the derangements consequent on a change of residence prevented my availing myself of that most favourable conjuncture, and it was not till the autumn of that year that the inquiry could be resumed. From that time to the present date it has been prosecuted at intervals as the weather would allow, though owing to the almost unprecedented continuance of bad weather during the whole of the past sumner and autumn (184l), it has of late been almost wholly suspended $\neq$. In photographic processes, where silver and other metals are used, the effect of

* From the Philosophical Transactions, 1842, p. 181 ; having been received by the Royal Society June 15, and read June 16, 1842.

+ The paragriphs, for convenience of reference, are numbered in continuation of those of the previous paper referred to in the text [an abstract of which will be found in Phil. Mag. S.3, vol. xvi. p. 331.-EDit.].

$\ddagger$ This was written in April 1842, since which a repetition of the season of 1840 seems to have commenced. 
light is so rapid that the state of the weather, as to gloom or sunshine, is of little moment. It is otherwise in the class of photographic actions now to be considered, in which exposure to the concentrated spectrum for many hours, to clear sunshine for several days, or to dispersed light for whole months, is requisite to bring on many of the effects described, and those some of the most curious. Moreover, in such experiments, when unduly prolonged by bad weather, the effects due to the action of light become mixed and confounded with those of spontaneous changes in the organic substances employed, arising from the influence of air, and especially of moisture, \&c., and so give rise to contradictory conclusions, or at all events preclude definite results, and obscure the perception of characters which might serve as guides in an intricate inquiry, and afford hints for the conduct of future experiment. It is owing to these causes that $I$ am unable to present the results at which I have arrived, in any sort of regular or systematic connexion; nor should $I$ have ventured to present them at all to the Royal Society, but in the hope that, desultory as they are, there may yet be found in them matter of sufficient interest to render their longer suppression unadvisable, and to induce others more favourably situated as to climate, to prosecute the subject.

150. The materials operated on in these experiments have been for the most part the juices of the flowers or leaves of plants, expressed, either simply, or with addition of alcohol, or under the influence of other chemical reagents. Some few resinous and dyeing substances have also been subjected to experiment, but with less perseverance than the obvious practical importance of this branch of the subject might demand, except in the case of guaiacum, whose relations to light, heat, and chemical agents are exceedingly remarkable and instructive, for which reason, as well as because some of these relations have been treated of in my former paper, I shall commence the account of my later experiments with those made on this substance. But in the first place it is necessary to state that the apparatus used for forming, concentrating, and fixing the spectrum, was the same with that described in Art. 67. of that paper; the prism being that of flint-glass by Fraunhofer, there mentioned; the area of the section of the incident sunbeam $=1.54$ square inch, and the dimensions of the principal elements of the luminous spectrum, identical with those recorded in $\$ .70$, so that the following results, when numerically stated (in measures of which the unit is one-thirtieth of an inch), will be comparable with those previously described. To spare reference, however, it may be here mentioned that the diameter of the sun's image in the focus of the achro- 
matic lens used is 7.20 of such thirtieths; and that the extent of the visible spectrum corrected for the sun's semidiameter at either end, equals 53.92 thirtieths, of which 13.30 are considered as reckoned negatively to the extreme visible red from a fiducial point or centre corresponding to the mean yellow ray ; and $40^{\circ} 62$ positively, from the same centre to the terminal violet, both as seen through a certain standard blue glass, which lets both extremes pass freely and insulates the mean yellow with considerable precision. The correction for the sun's semidiameter has been applied in what follows to all measures up to terminations of spectra, unless where the contrary is expressed. Maxima and minima of action, and neutral points neither require nor admit this correction.

\section{Guaiacum.}

151. A solution of this resin in alcohol, spread evenly on paper, gives a nearly colourless ground. A slip of this paper exposed to the spectrum is speedily impressed with a fine blue streak over the region of the violet rays, and far beyond, as described in Art. 92. If the paper during this action be carefully defended from extraneous light, this is the only perceptible effect; but if dispersed light be admitted, the general ground of the paper is turned to a pale brownish green, with exception of that portion on which the less refrangible rays fall, which, by their agency, is defended from the action of the dispersed light and preserves its whiteness, as in the case of the argentine paper described in Art. 60. The spectrum, therefore, ultimately impressed, consists of two portions similar to those described in Art. 93, and of nearly the same extent, that is to say, a white or pale yellowish portion having its maximum of intensity at 0.0 , and extending from -11.9 (corrected for the sun's semidiameter) to $+12 \cdot 0$, or thereabouts, at which point the character of the action changes, and a blue, of a somewhat smoky gray cast, commences, which attains a maximum at $+40 \%$, thence degrades to an intermediate minimum at $+47{ }^{\circ}$, attains a second and much stronger maximum at $+61 \cdot 0$, and ceases at $72 \cdot 4$. The precise numbers vary materially in different specimens and with the length of exposure. The type of this spectrum, of its natural length, is represented in Plate I. fig. 1, in which the abscissa being measured along the length of the spectrum, from the fiducial centre $\mathrm{Y}$ both ways, the ordinates express the intensities of photographic action at each corresponding point, as estimated from the amount of colour induced or prevented. In this type the portion corresponding to the less refrangible rays is represented by negative values of the or- 
dinate agreeably to Art. 93 , where it is shown that these rays not only prevent the blue colour from being produced by the more refrangible ones, but destroy it when so produced. Another specimen gave the following dimensions: $\mathrm{Y} a=-$ $11 \cdot 4 \mathrm{Y} b=-9 \cdot 5, \mathrm{Y} c=+30 \cdot 0, \mathrm{Y} d=+61 \cdot 0, \mathrm{Y} e=+80^{\circ} \cdot 4$, and this is the greatest extent of action I have hitherto observed.

152. A portion of the same paper was exposed, dry, to an atmosphere of chlorine considerably diluted with common air, which imparted to it a pale, dirty, greenish yellow hue. Being thence transferred immediately to the spectrum, the result was not a little remarkable. The whole spectrum, the green excepted, was impressed in faint tints nearly corresponding to the natural ones. The red was evident-the yellow dilute and nearly white - the blue a fine sky-blue, while beyond the violet succeeded a train of somewhat greenish darkness. These tints proved fugitive, and in twenty-four hours were nearly obliterated.

153. When paper fresh washed with tincture of guaiacum and still wet is exposed to chlorine, it instantly acquires a fine and full Prussian blue colour, which however passes speedily to brown if the action be prolonged. The colour is difficult to preserve in its full intensity, and fades considernbly in drying, becoming at the same time somewhat greenish. Exposed wet to the spectrum, it is found to have become much more sensitive, and is inmediately attacked with great energy by the red rays, which destroy the blue colour, converting it to a brownish or reddish yellow. The action extends rapidly up the spectrum as far as the extreme violet, in which ray, however, the tint impressed or left undestroyed passes to a hue partaking of violet, and indicating by the change what ought probably to be regarded as a neutral point at $+12 \cdot 0$. The impressed spectrum (corrected for semidiameter) commences at $a$, fig. 2 , at $-13 \cdot 4$; the maximum $b$ of the positive action occurs at $-9 \cdot 0$, the neutral point $c$ at $+12 \cdot 0$, the maximum $d$ of negative action at $+33 \cdot 0$, and the sensible termination $e$ of the impression at $+60^{\circ} 0$.

154. The action of gaseous chlorine is too energetic to be easily arrested at the proper point, besides which this gas also acts powerfully on the alcohol employed. To obviate these inconveniences, paper thoroughly impregnated with guaiacum by washing with the tincture, and drying in a gentle heat, was steeped in weak aqueous solution of chlorine, by which process it slowly acquired a beautiful and pure celestial blue colour. It is very sensitive, and may be conveniently used for copying engravings, \&c., which it does with this singularity, 
that the picture penetrates the paper and appears on the back of very nearly the same intensity as on the face*. Indeed, if the picture be over-sunned the back will exhibit a perfect impression, while the face is spoiled, which produces a very strange effect: exposed to the spectrum, the blue colour is converted to a pale reddish yellow in the region of the less refrangible rays, and simply whitened in the more refrangible region. The action, when prolonged till the light seems to have no further influence, extends from $-12 \cdot 4$, corrected for semidiameter, to +40 , or thereabouts, where it dies away insensibly. The maximum of photographic action occurs at $-8 \cdot 7$, and some trace of a minimum is perceptible at $+11 \cdot 5$. Photographs taken on this paper, or spectra impressed on it, are fugitive-lose much of their force and beauty in a few days, and at length vanish altogether.

155. When paper is washed with a solution of guaiacum in soda it acquires a green colour, though the solution itself is brown. By inclining the paper and carrying the wash always from below upwards, a very even tint may be obtained. The excess of liquid being blotted off, aqueous solution of chlorine was poured over it (on a slope) till all the alkali was saturated, and the liquid ran off smelling strongly of chlorine. Thus was produced a paper (No. 1168.) very evenly tinted, and varying in colour from a deep, somewhat greenish, to a fine celestial blue, according to the strength of the solutions employed. It is very sensitive, and is attacked with especial energy by rays in the spectrum, ranging from $-11 \cdot 4$ to $+11 \cdot 4$ with a maximum at $-9 \cdot 0$, the type being as in fig. 3 .

156. When paper so prepared is exposed, wet, to a temperature of $212^{\circ}$ Fahr., it is immediately discoloured, the green changing to a sere or brownish yellow. The same change is produced after some little time at a temperature of $190^{\circ}$, and still more slowly, though yet completely, at $180^{\circ}$. At $175^{\circ}$ the discoloration is incomplete and very slow; and below that temperature the colour is not affected. If the paper be perfectly dried in a temperature gradually raised to $212^{\circ}$, the discoloration requires a considerably higher temperature, ranging from $220^{\circ}$ to $275^{\circ}$, according to the time of exposure, being very slow at the former limit and almost immediate at the latter. These changes are independent of the action of light, being produced under mercury.

157. The destruction by heat of the green or blue colour superinduced on guaiacum by the more refrangible rays of light, was noticed by Wollaston, and it would seem, on a

* For another remarkable case of this kind see the Postscript to this paper. 
consideration of his experiments and of those described in the last article, that nothing further is requisite for operating the change from the green or blue to the yellow state, than the assumption of a certain temperature dependent on its state of dryness, and varying according to that state between the limits of $180^{\circ}$ and $280^{\circ}$. Nevertheless, if we consider that the same change is produced by rays of the spectrum which are very far from being the hottest, while yet the extra-spectral thermic rays, under precisely the same circumstances of exposure, produce no such effect, though far surpassing in mere calorific power those which do, we shall see reason to doubt the sufficiency of this vier of the matter. The following experiments were therefore instituted with a view to its further elucidation.

158. A slip of the paper No. 1168 was moistened and subjected in clear sunshine to the action of the spectrum. The colour was discharged from the region occupied by the less refrangible luminous rays, as described in Art. 155. At the same time, the more distant thermic rays beyond the spectrum produced their proper effect, in evaporating the moisture from those portions on which they fell; so that in due time the heat-spots $\delta$ and $\gamma$ became apparent (see Art. 136.), the former very distinctly, the latter perceptibly. The spot $\beta$ (which is remarkable) was scarcely if at all formed. So long then as the paper continued moist and remained under the influence of the thermic rays, the appearances were those of $a$ diminution of colour (Art. 131.), operated by the thermic rays $\delta$ and $\gamma$. But the discoloration in these points was only apparent, for as the paper dried these heat-spots disappeared, leaving its colour quite unchanged at those points; while the photographic impression really produced within the visible spectrum, remained and went on increasing in intensity. The non-luminous thermic rays, therefore, though clearly shown to have been active as heat, were yet incapable of effecting that peculiar chemical change which other rays much less copiously endowed with heating power, were all the while producing.

159. It may be objected to this, that no proof is afforded in the above-related experiment, that any part of the paper actually attained a temperature of $180^{\circ}$ or more; that in conm sequence no discoloration due to the action of heat (quoad heat) was produced; and that the discoloration which did take place was sui generis, and originated with the light and not the heat of that part of the spectrum to which it corresponded. A slip of the same paper (1168.) was therefore exposed dry to the spectrum in such a way to to leave its back accessible; and an iron heated below redness was then ap- 
proached to it so as just not to discolour the paper. Under such circumstances it might be expected that the additional heat thrown on the paper in the region of the thermic rays would turn the scale in their favour at their points of greatest intensity, and give ocular proof of their action by a decided discharge of colour at those points. But no such result was obtained, nor could I succeed in rendering visible any of the heat-spots $\alpha, \beta, \gamma, \delta$, even when the heated iron was brought so near as to produce a commencement of discoloration over the whole of that region of the paper where they ought to have shown themselves.

160. On the other hand, a remarkable, but by no means an unexpected influence, was exercised by the heat so thrown on that part of the paper where the less refrangible rays fell, and where the discoloration was in progress under their agency. For it was observed that, under these circumstances, the discoloration in question went on with much greater rapidity, so much so indeed, that the same amount of it, which without extraneous heat would have required twenty ninutes or half an hour's exposure to the spectrum to produce, was now produced in two or three minutes. Obscure terrestrial heat, therefore, is shown to be capable of assisting and being assisted in operating this peculiar change, by those rays of the spectrum, whether luminous or thermic, which occupy its red, yellow, and green regions; while on the other hand it receives no such assistance from the purely thermic rays beyond the spectrum, acting under precisely similar circum. stances, and in an equal state of condensation.

161. When heat was similarly applied by radiation from behind, and from a non-luminous source, over the more refrangible region of a spectrum thrown on paper simply washed with tincture of guaiacum and not previously blued either by chlofine or by light, the blue colour induced in the more refrangible rays was still produced, and of the same tint in the same points as if no heat had acted. This effect, the contrary to what the previous experiment would have led to expect, shows how little any reasonings on these points enable us at present to anticipate experience.

162. The discharge of colour from blued guaiacum by mere heat, has been shown above (Art. 156.) to take place at a much lower temperature in the presence of moisture than when dry; and a similar destruction of colour, under similar circumstances, takes place with many other vegetable preparations. Paper, for instance, coloured with the juice of the Viola tricolor (Art. 90.), is speedily whitened in the dark, while wet, by the heat of boiling water, though dry heat does 
not affect it. And under the action of the spectrum it is discoloured (though much more slowly) by the same, or nearly the same rays which are effective in the case of guaiacum. The colour of paper tinged with the juice of the common red stock is not affected when dry by any heat short of what suffices to scorch the paper, but when wet (as when exposed to steam) it is speedily discharged. There are few, if any vegetable colours indeed which long resist the combined effects of heat and moisture, even when light is excluded, still less when admitted*.

\section{Of the Colours of Flowers in general under the action of the Spectrum.}

163. In operating on the colours of flowers I have usually proceeded as follows:- the petals of the fresh flowers, or rather such parts of them as possessed a uniform tint, were crushed to a pulp in a marble mortar, either alone, or with addition of alcohol, and the juice expressed by squeezing the pulp in a clean linen or cotton cloth. It was then spread on paper with a flat brush, and dried in the air without artificial heat, or at most with the gentle warmth which rises in the ascending current of air from an Arnott stove. If alcohol be not added, the application on paper must be performed immediately, since exposure to the air of the juices of most flowers (in some cases even for but a few minutes) irrecoverably changes or destroys their colour. If alcohol be present this change does not usually take place, or is much retarded; for which reason, as well as on account of certain facilities afforded by its admixture in procuring an even tint (to be presently stated), this addition was commonly, but not always made.

164. Most flowers give out their colouring matter readily enough, either to alcohol or water. Some, however, as the Eschnolzias and Calceolarias, refuse to do so, and require the addition of alkalies, others of acids, \&c. When alcohol is added, it should, however, be observed that the tint is often, apparently, much enfeebled, or even discharged altogether, and that the tincture, when spread on paper, does not reappear of its due intensity till after complete drying. 'The temporary destruction of the colour of the blue heartsease by alcohol has been noticed in my former paper (Art. 90.), nor is

* On the effects of light, air, and moisture at common temperatures, as discolouring agents on several dyeing materials, I may refer to M.Chevreul's elaborate memoir ( $A c a d$. $R$. des Sciences, tom. xvi.). M. Chevreul's experiments, however, relate to the action of light simply as it comes from the sun without prismatic separation, and have therefore little or nothing in common with the objects of this paper. 
that by any means a singular instance. In some, but in very few cases, it is destroyed, so as neither to reappear on drying, nor to be capable of revival by any means tried. And in all cases long keeping deteriorates the colours and alters the qualities of the alcoholic tinctures themselves, so that they should always be used as fresh as possible.

165. If papers tinged with vegetable colours are intended to be preserved, they must be kept perfectly dry and in darkness. A close tin vessel, the air of which is dried by quicklime (carefully enclosed in double paper bags, well pasted at the edges to prevent the dust escaping), is useful for this purpose. Moisture (as already mentioned, especially assisted by heat) destroys them for the most part rapidly, though some (as the colour of the Senecio splendens) resist obstinately. Their destructibility by this agency, however, seems to bear no distinct relation to their photographic properties.

166. This is also the place to observe that the colour of a flower is by no means always, or usually, that which its ex pressed juice imparts to white paper. In many cases the tints so imparted have no resemblance to the original hue. Thus, to give only a few instances, the red damask rose of that intense variety of colour, commonly called by florists the Black Rose, gives a dark slate blue, as do also the clove carnation and the black holyoak; a fine dark brown variety of Sparaxis gave a dull olive green; and a beautiful rose-coloured tulip, a dirty bluish green; but perhaps the most striking case of this kind is that of a common sort of red poppy (Papaver Rheum?), whose expressed juice imparts to paper a rich and most beautiful blue colour, whose elegant properties as a photographic material will be further alluded to hereafter*.

167. This change of colour is probably owing to different causes in different flowers. In some it undoubtedly arises from the escape of carbonic acid, but this as a general cause for the change from red to blue, has, I am aware, been controverted $t$. In some (as is the case with the yellow Ranunculi) it seems to arise from a chemical alteration depending on absorption of oxygen; and in others, especially where the expressed juice coagulates on standing, to a loss of vitality or disorganization of the molecules. The fresh petal of a single flower, merely crushed by rubbing on dry paper, and instantly dried, leaves a stain much more nearly approximating to the original hue. This, for example, is the only way in

* A semi-cultivated variety was used, having dark purple spots at the bases of the petals. The common red poppy of the chalk (Papaver hybridum) gives a purple colour much less sensitive and beautiful.

it Nicholson's Juurnal, 
which the fine blue colour of the common field Veronica can be imparted to paper. Its expressed juice, however, quickly prepared, when laid on with a brush, affords only a dirty neutral gray, and so of many others. But in this way no even tint can be had, which is a first requisite to the experiments now in question, as well as to their application to photography.

168. To secure this desirable evenness of tint, the following manipulation will generally be found successful. The paper should be moistened at the back by sponging and blotting off. It should then be pinned on a board, the moist side downwards, so that two of its edges (suppose the right-hand and lower ones) shall project a little beyond those of the board. The board being then inclined twenty or thirty degrees to the horizon, the alcoholic tincture (mixed with a very little water, if the petals themselves be not very juicy) is to be applied with a brush in strokes from left to right, taking care not to go over the edges which rest on the board, but to pass clearly over those which project, and observing also to carry the tint from below upwards by quick sweeping strokes, leaving no dry spaces between them, but keeping up a continuity of wet surface. When all is wet, cross them by another set of strokes from above downwards, so managing the brush as to leave no floating liquid on the paper. It must then be dried as quickly as possible over a stove, or in a current of warm air, avoiding, however, such heat as may injure the tint. 'The presence of alcohol prevents the solution of the gummy principle, which, when present, gives a smeary surface; but the evenness of tint given by this process results chiefly from that singular intestine movement which always takes place when alcohol is in the act of separation from water by evaporation-a movement which disperses knots and blots in the film of liquid with great energy, and spreads them over the surrounding surface.

169. The action of the spectrum, or of white light, on the colours of flowers and leaves, is extremely various, both as regards its total intensity and the distribution of the active rays over the spectrum. But certain peculiarities in this species of action obtain almost universally.

1st. The action is positive, that is to say, light destroys colour; either totally, or leaving a residual tint, on which it has no further, or a very much slower action. And thus is effected a sort of chromatic analysis, in which two distinct elements of colour are separated, by destroying the one and leaving the other outstanding. The older the paper, or the tincture with which it is stained, the greater is the amount of this residual tint. 
2nd. The action of the spectrum is confined, or nearly so, to the region of it occupied by the luminous rays, as contradistinguished both from the so-called chemical rays, beyond the violet, which act with the chief energy on argentine compounds, but are here for the most part ineffective, on the one hand, and on the other, from the thermic rays beyond the red, which appear to be totally so. Indeed, I have hitherto observed no instance of the extension of this description of photographic action on vegetable colours beyond, or even quite up to, the extreme red.

170. Besides these, it may also be observed that the rays effective in destroying a given tint, are, in a great many cases, those whose union produces a colour complementary to the tint destroyed, or at least one belonging to that class of colours to which such complementary tint may be referred. For example, yellows tending towards orange are destroyed with more energy by the blue rays; blues by the red, orange, and yellow rays; purples and pinks by yellow and green rays.

171. These are certainly remarkable and characteristic peculiarities, and must indeed be regarded as separating the luminous rays by a pretty broad line of chemical distinction from the non-luminous; though whether they act as such, or in virtue of some peculiar chemical quality of the heat which accompanies them as heat, is a point which the experiments on guaiacum, above described, seem to leave rather equivocal. In the latter alternative, chemists must henceforward recognize differences not simply of intensity, but of quality in heat from different sources; of quality, that is to say, not merely as regards degree of refrangibility or transcalescence, but as regards the strictly chemical changes it is capable of effecting in ingredients subjected to its influence.

172. As above stated, these peculiarities, at least the first two, obtain almost universally. Exceptions, however, though very rare, do occur, as will be more particularly mentioned hereafter. 'The third rule is much less general, and is to be interpreted with considerable latitude; but among its exceptions I have been unable to detect any common principle capable of being distinctly enunciated.

173. Lastly, it requires to be expressly mentioned, that the habitudes of the colours, both of the flowers and leaves of plants, with relation either to white light or to the prismatic rays, vary materially with the advance of the season, and perhaps also with the hour of the day at which they are gathered. Generally speaking, so far as 1 have been able to observe, the earlier flowers of any given species reared in the open air (provided they are well ripened, i. e. the colour fully deve- 
loped) are more sensitive than those produced even from the same plant, at a late period in its flowering, and have their colours more completely discharged by light. As the end of the flowering period comes on, not only the destruction of the colour by light is slower, but residual tints are left which resist obstinately. A very remarkable case of this kind was noticed in Chryseis californica, the earliest flowers of which exhibited in the photograph of their spectrum a well-insulated round spot, eaten away by red rays almost at its extremity, which spot I never was able to reproduce with later flowers from the same root. Those gathered at the end of its flowering also left a residual yellow of extreme obstinacy*, which was by no means the case with the earlier flowers.

174. It would be waste of time to enumerate all the vegetable tints which I have subjected to experiment, comprising most of the ordinary hardy garden and wild flowers of the country. 'To the rarer and more splendid species which adorn the stoves and greenhouses of florists, I have had little access, a circumstance $I$ much regret, and which leads me to take this opportunity of mentioning, that specimens of paper stained with the juices of highly-coloured, or otherwise remarkable flowers or leaves, either by alcoholic extraction, or by simple expression (if accompanied with the botanical name of the plant used), will be highly acceptable, from whatever quarter received. I shall here set down only those which afforded some ground for special remark, so far as I have yet pushed the inquiry.

\section{Colours of particular Flowers.}

175. Corchorus Japonica.-The flowers of this common and hardy but highly ornamental plant, are of a fine yellow, somewhat inclining to orange, and this is also the colour the expressed juice imparts to paper. As the flower begins to fade the petals rohiten, an indication of their photographic sensi-. bility, which is amply verified on exposure of the stained paper to sunshine. I have hitherto met with no vegetable colour so sensitive. If the flowers be gathered in the height of their season, paper so coloured (which is of a very even and beautiful yellow) begins to discolour in ten or twelve minutes in clear sunshine, and in half an hour is completely whitened. 'The colour seems to resist the first impression of the light, as if by some remains of vitality, which being overcome, the tint gives way at once, and the discoloration when commenced

* Probably, therefore, useful in dyeing. The species is that most commonly cultivated in gardens, with bright yellow petals having orange-coloured bases. 
goes on rapidly. It does not even cease in the dark when once begun. Hence it happens that photographic impressions taken on such paper, which when fresh are very sharp and beautiful, fade by keeping, visibly from day to day, however carefully preserved from light. Specimens of such photographs (copies of engravings) are submitted with this paper for inspection. They require from half an hour to an hour to complete, according to the sunshine. Hydriodate of potash cautiously applied, retards considerably, but does not ultimately prevent, this spontaneous discharge.

176. Exposed to the spectrum, in about fifteen or twenty minutes the colour is totally destroyed and the paper whitened in the whole region of the green, blue and violet rays, to which therefore the most energetic action is confined, agreeably to the law of complementary tints (Art. 170.). If the action of the spectrum be prolonged, a much feebler whitening becomes sensible in the red, and a trace of it also beyond the violet into the "lavender" rays. In this state the type of the impressed spectrum (in an experiment made on the 7th of April in the present year) was as in fig. 4, indicating three obsolete maxima $c, d, e$, and a very sudden diminution of the action at $b, f$, the dimensions being as follows: $\mathrm{Y} a=-9 \cdot 4, \mathrm{Y} b$ $=+7 \cdot 1, \mathrm{Y} c=+12 \cdot 5, \mathrm{Y} d=+23 \cdot 5, \mathrm{Y} e=+34 \cdot 0, \mathrm{Y} f$ $=+41 \cdot 4, Y g=+59 \cdot 7$. The paper thus impressed was again re-examined on the 2nd of May, or after twenty-five days, during which interval it had been exposed to free air, but only to feeble and dispersed occasional lights. It was found to have undergone a remarkable change, two distinct white spots having become insulated, or nearly so, at the very extremities of the impressed spectrum, the three maxima above indicated having also become much more distinct, and two new, subordinate ones, having begun to show themselves in the faint traces connecting the spots above mentioned with the main impression. The type of the spectrum in this state was as represented in fig. 5 , and the places of the several maxima being as follows: $-1 \mathrm{st},-10.0 ; 2 \mathrm{nd},-0.5 ; 3 \mathrm{rd},+12.0$; 4 th, $+29 \cdot 0 ; 5$ th,$+40 \cdot 0 ; 6$ th $,+50:: ; 7$ th,$+61 \cdot 0$. The terminal spot at the red extremity was nearly equal in diameter to the sun's image; that at the least refracted end, corresponding in place to rays much beyond the last violet, was smaller, but perfectly distinct, and as it constitutes the only instance $I$ have yet encountered of a definite ray in this region of the spectrum*, 1 have been thus particular in describing the phænomenon.

- Since this was written, other cases, extremely remarkable, among the argentine preparations, have presented themselves. See Art. 214.

Phil. Mag. S. 3. Vol. 22. No. 142. Jan. 1843. 
177. Common ten-reeks Stock, Mathiola annua.-The colour imparted by the petals of the double variety of this flower* to alcohol (at least when spread on paper, for it is in great measure dormant in the liquid tincture) is a rich and florid rose-red, varying, however, from a fiery tint almost amounting to scarlet, on the one hand, to a somewhat crimson or slightly purplish red on the other, according to the accidents of its preparation, or the paper used. When fresh prepared it is considerably sensitive, an hour or two of exposure to sunshine being sufficient to produce a sensible discoloration, and two or three days entirely to whiten it. This quality is greatly deteriorated by keeping, but papers prepared with it even after eight or ten months, still with patience yield extremely beautiful photographs, several specimens of which in various states of the tincture are submitted for inspection to the meeting. Exposed to the spectrum, the rays chiefly active in operating the discoloration are found to be those extending from the yellow to the less refrangible red, beyond which rays the action terminates abruptly. A Above the yellow it degrades rapidly to a minimum in the blue, beyond which it recovers somewhat, and attains a second but much feebler maximum in the violet rays.

178. Paper stained with the tincture of this flower is changed to a vivid scarlet by acids, and to green by alkalies; if ammonia be used the red colour is restored as the ammonia evaporates, proving the absence of any acid quality in the colouring matter sufficiently energetic to coerce the elastic force of the alkaline gas. Sulphurous acid whitens it, as do the alkaline sulphites; but this effect is transient, and the red colour is slowly restored by free exposure to air, especially with the aid of light, whose influence in this case is the more remarkable, being exactly the reverse of its ordinary action on this colouring principle, which it destroys irrecoverably, as above stated. The following experiments were made to trace and illustrate this curious change.

179. Two photographic copies of engravings taken on paper tinted with this colour were placed in a jar of sulphurous acid gas, by which they were completely whitened, and all traces of the pictures obliterated. They were then exposed to free air, the one in the dark, the other in sunshine. Both recovered, but the former much more slowly than the latter. The restoration of the picture exposed to the sun was completed in

* That imparted by the single flowers is very much less sensitive, as is also that of the dull red or purplish variety, whether double or single. The most florid red double flowers, in the height of their fiowering, yield the best colour. 
twenty-four hours, that in the dark not till after a lapse of two or three days.

180. A slip of the stained paper was wetted with liquid sulphurous acid and laid on blotting-paper similarly wetted. Being then crossed with a strip of black paper, it was laid between glass plates and (evaporation of the acid being thus prevented) was exposed to full sunshine. After some time the red colour (in spite of the presence of the acid) was considerably restored in the portion exposed, while the whole of the portion covered by the black paper remained (of course) perfectly white.

181. Slips of paper, stained as above, were placed under a receiver, beside a small capsule of liquid sulphurous acid. When completely discoloured they were subjected (on various occasions, and after various lengths of exposureto the acid fumes from half an hour to many days) to the action of the spectrum; and it was found, as indeed I had expected, that the restoration of colour was operated by rays complementary to those which destroy it in the natural state of the paper, the violet rays being chiefly active, the blue almost equally so, the green little, and the yellow, orange, and most refrangible red not at all. In one experiment a pretty well-defined red solar image was developed by the least refrangible red rays also, being precisely those for which in the unprepared paper the discolouring action is abruptly cut off. But this spot I never succeeded in reproducing; and it ought also to be mentioned, that, according to differences in the preparation not obvious, the degree of sensibility, generally, of the bleached paper to the restorative action of light differed greatly ; in some cases a perceptible reddening being produced in ten seconds, and a considerable streak in two minutes, while in others a very long time was required to produce any effect.

182. The dormancy of this colouring principle, under the influence of sulphurous acid, is well shown by dropping a little weak sulphuric acid on the paper bleached by that gas, which immediately restores the red colour in all its vigour. In like manner alkalies restore the colour, converting it at the same time into green.

183. Papaver orientale. - The chemical habitudes of the sulphurous acid render it highly probable that its action, in inducing a dormant state of the colorific principle, consists in a partial deoxidizement, unaccompanied however with disorganization of its molecules. And this view is corroborated by the similar action of alcohol already spoken of; similar, that is, in kind, though less complete in degree. Most commonly, vegetable colours, weakened by the action of alcohol, are 
speedily restored on the total evaporation of that ingredient. But one remarkable instance of absolute dormancy induced by that agent, has occurred to me in the case of the Papaver orientale, a flower of a vivid orange colour, bordering on scarlet, the colouring matter of which is not extractable otherwise than by alcohol, and then only in a state so completely masked, as to impart no more than a faint yellowish or pinkish hue to paper, which it retains when thoroughly dry, and apparently during any length of time without perceptible increase of tint. If at any time, however, a drop of weak acid be applied to paper prepared with this tincture, a vivid scarlet colour is immediately developed, thus demonstrating the continued though latent existence of the colouring principle. On observing this, it occurred to me to inquire whether, in its dormant state, that principle still retained its susceptibility of being acted on by light, since the same powerful and delicate agent, which had been shown in so many cases as to constitute a general law, capable of disorganising and destroying vegetable colours actually developed, might easily be presumed competent to destroy the capacity for assuming colour, in such organic matter as might possess it, under the influence of their otherwise appropriate chemical stimuli. A strip of the paper was therefore exposed for an hour or two to the spectrum, but without any sensible effect, the whole surface being equally reddened by an acid. As this experiment sufficiently indicated the action of light, if any, to be very slow, I next placed a strip, partly covered, in a south-east window, where it remained from June 19 to August 19, receiving the few and scanty sunbeams which that interval of the deplorable summer of 1841 afforded. When removed, the part exposed could barely be distinguished from the part shaded, as a trifle yellower. But on applying acid, the exposed and shaded portions were at once distinguished by the assumption of a vivid red in the latter, the former remaining unchanged.

184. A mezzotinto picture was now pressed on a glazed frame over another portion of the same paper, and abandoned on the upper shelf of a green-house to whatever sun might occur from August 19 to October 19. The interval proved one of almost uninterrupted storm, rain, and darkness. On removal, no appearance whatever of any impressed picture could be discerned, nor was it even possible to tell the top of the picture from the bottom. It was then exposed in a glass jar to the fumes of muriatic acid, when, after a few minutes, the development of the dormant picture commenced, and slowly proceeded, disclosing the details in a soft and pleasing style. Being then laid by in a drawer, with free access of air, 
the picture again faded, by very slow degrees, and on January 2 , 1842, was found quite obliterated. Being then again subjected to the acid vapour, the colour was reproduced. How often this alternation might have gone on I cannot say, the specimen having been mislaid or destroyed. But a portion of such paper photographically impressed with a stamped pattern, accompanies this communication for the satisfaction of any member who may wish to try the experiment. The extreme slowness of the action precludes any prismatic analysis of the process, and it cannot be too often repeated that the use of coloured glasses in such inquiries serves only to mislead. Of dormant photographic impressions generally, whether slowly developing themselves by lapse of time, or at once revivable by stimuli, as well as of the spontaneous fading and disappearance of such impressions, I shall have more to say hereafter, having encountered several very curious cases of the kind in studying the habitudes of gold, platina, \&c. I would here only observe, that a consideration of many such phænomena has led me to regard it as not impossible that the retina itself may be photographically impressible by strong lights, and that some at least of the phanomena of visual spectra and secondary colours may arise from the sensorial perception of actual changes in progress in the physical state of that organ itself, subsequent to the cessation of the direct stimulant.

[To be continued.]

III. A Reply to Professor Kelland's Observations in the Philosophical. Magazine for November 1842. By the Rev. M. O'Brien *.

A S my final reply to Professor Kelland, I beg to state very $A$ briefly the following facts :-

1st. Professor Kelland has given no answer to the questions I asked him, viz. "W Why did he bring forward an expression for $v^{2}$ from his ' Theory of Heat' as equivalent to mine, when it most clearly does not account for dispersion independently of the hypothesis of finite intervals?" And again, "Why did he not quote the words which follow this expression in bis ' Theory of Heat,' containing his own admission that it was too uncertain to be made use of ?". These questions contain my chief "charges" against Professor Kelland; and since he has not answered them, I conclude that he cannot deny his attempt to create an impression that he had, in his "Theory of Heat,' anticipated my explanation of dispersion.

* Communicated by the Authcr. 

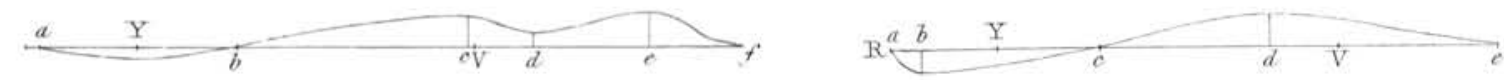

Fig 3 Art 1.55

Fig. 1 Art.zo

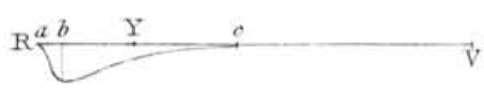

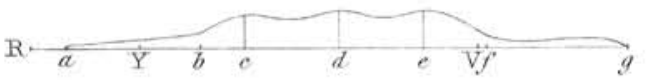

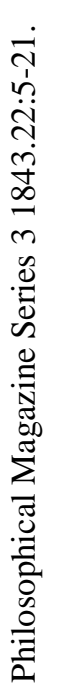
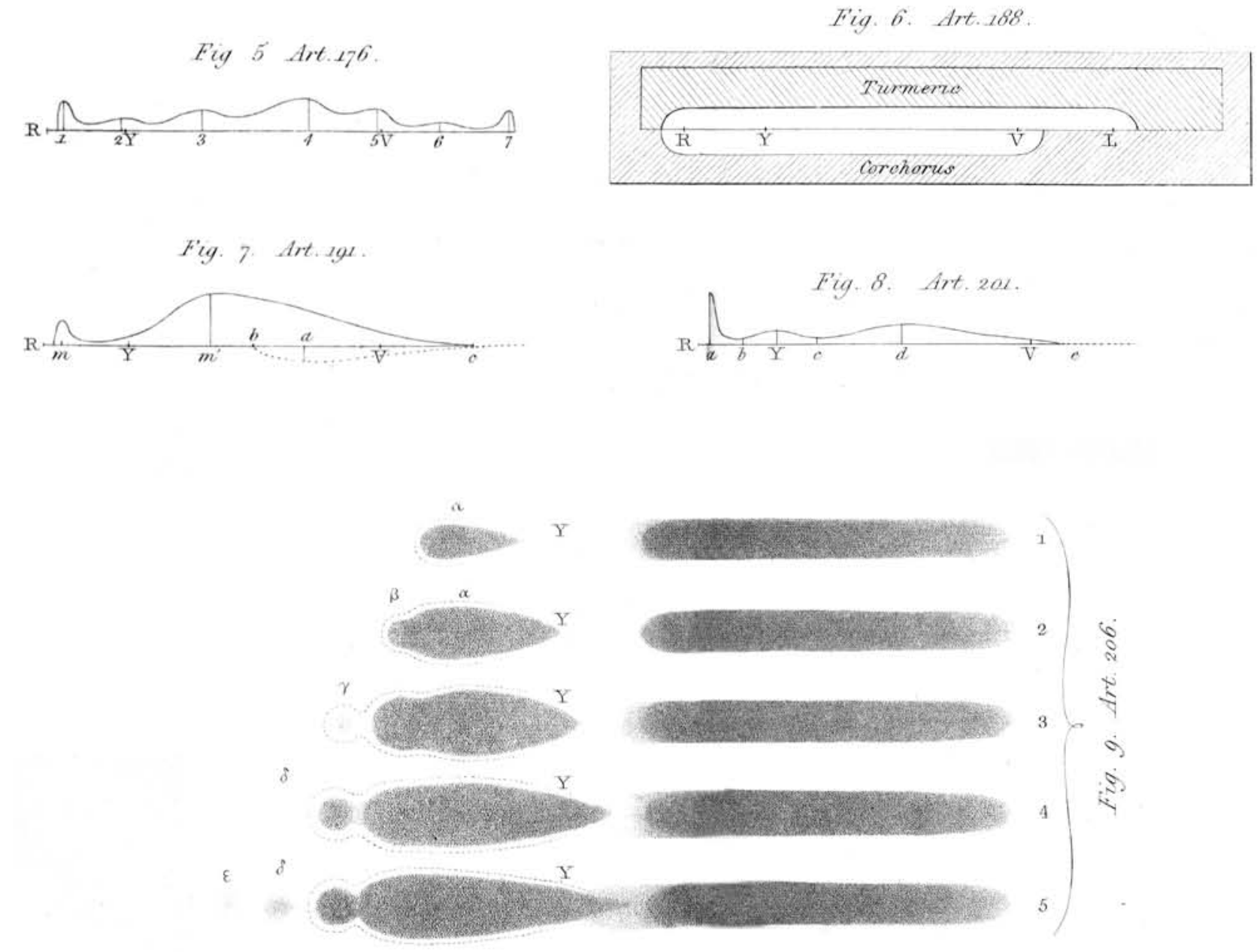

Thermographic Impressions.

Photographic Impressions.
Fig. 12. Art. 211

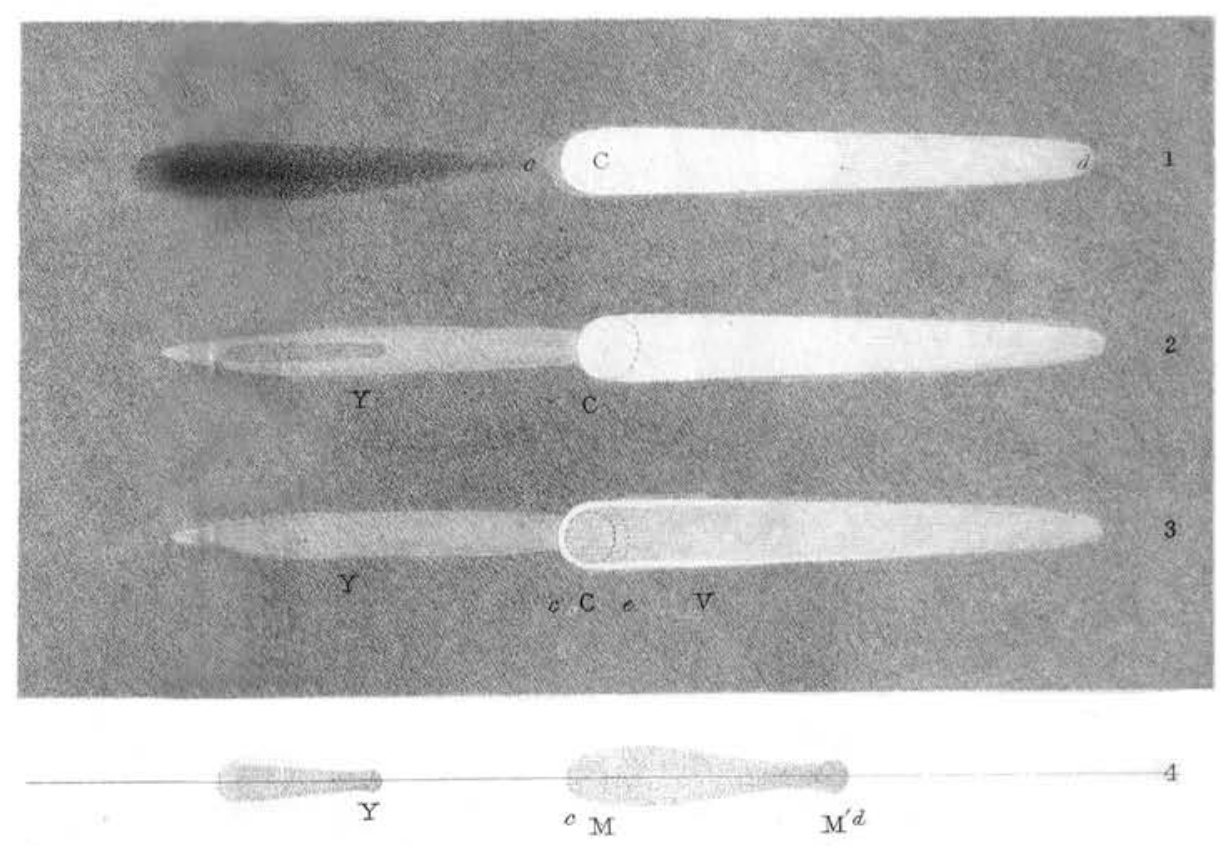

Fig. 20. Art. 207

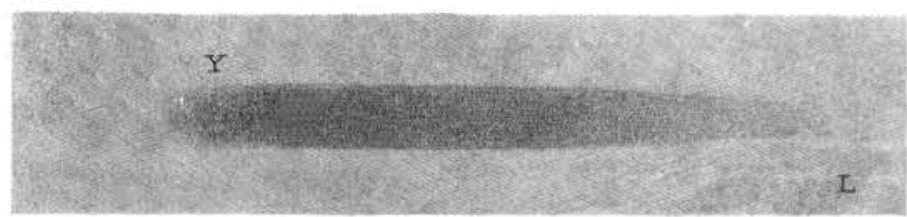

Fig. It. Art. 212

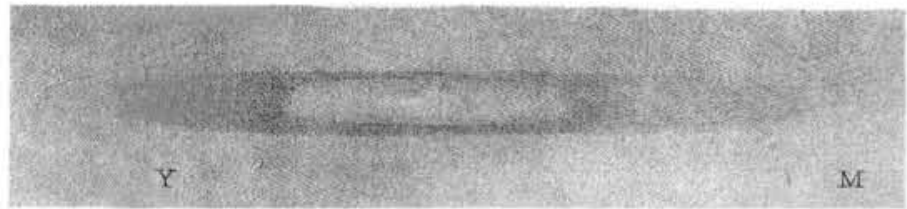


Thel Mag S. 3 Vol XXII Pr.2.

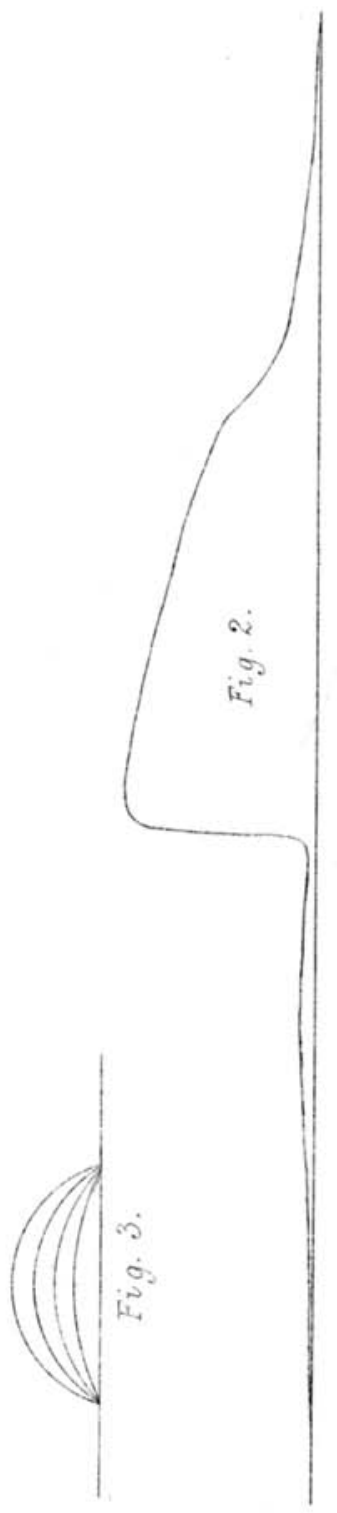

